\title{
Commentary: "Silver Nanoparticles Coated Poly(L-Lactide) Electrospun Membrane for Implant Associated Infections Prevention"
}

\author{
Selvaraj Vimalraj ${ }^{1,2}$ and Saravanan Sekaran ${ }^{2 *}$ \\ ${ }^{1}$ Centre for Biotechnology, Anna University, Chennai, India, ${ }^{2}$ Department of Pharmacology, Saveetha Dental College and \\ Hospitals, Saveetha Institute for Medical and Technical Sciences, Chennai, India
}

Keywords: PLLA fiber, guided bone regeneration (GBR), nanoparticle, silver, osteoblasts

\section{INTRODUCTION}

Jiaolong et al. (2020) published an interesting study "Silver Nanoparticles Coated Poly(L-Lactide) Electrospun Membrane for Implant-Associated Infections Prevention" on which I would like to express my viewpoints which may benefit other readers and researchers.

The guided bone regeneration (GBR) barrier membrane enhances the bone volume by allowing selective migration of estrogenic cells from boney margins and impeding other cells such as fibroblasts and epithelial cells from overlying mucosa in ridge augmentation procedures (Mellonig and Nevins, 1995). GBR membranes using resorbable and nonresorbable membranes can aid in treating moderate to severe bone defects. An FDAapproved PLLA polymer has been used clinically, and one of the common polymers is used in GBR procedures. Pure PLLA fibers often lack the suitability to support cell adhesion and proliferation diminishing its biological properties (Khatri et al., 2016). In addition to this, polymicrobial biofilm formation on the implanted material surface is the major reason for GBR membrane failure in vivo impeding its medical applications (Xie et al., 2010, Arciola et al., 2015, Willems et al., 2016; Trobos et al., 2018; Hong et al., 2019). To circumvent this limitation, the authors have utilized the intrinsic reduction property of polydopamine to ensure in situ synthesis of silver nanoparticles on PLLA surface imparting antimicrobial property. This study presents a new method in coating which may be utilized for coating many other nanoparticles and explored for bone tissue engineering applications.

This article was submitted to

Experimental Pharmacology and Drug Discovery,

a section of the journal

Frontiers in Pharmacology

Received: 16 August 2021 Accepted: 24 September 2021

Published: 28 October 2021

\section{HARNESSING THE PROPERTY OF PDA TO COAT VARIOUS METAL NANOPARTICLES ON PLLA}

Polydopamine is an interesting polymer, attributed to the presence of catechol moieties in its building blocks is similar to the mussel foot proteins have gained recent attention for grafting various biological agents (Ryu et al., 2010; Cui et al., 2012 Sun et al., 2014; Ding et al., 2016). Lee et al., 2007, reported self-polymerization of dopamine into thin films on a range of inorganic and organic materials by a simple dip-coating method. For instance, Liu et al., 2019 reported the coating of PLLA nanofiber scaffolds with osteogenic growth peptide via PDA coating enhanced cell adhesion, proliferation, osteogenic differentiation, and mineralization. PDA offers a simple, inexpensive, and versatile route compared with conventional surface modification methods in bone tissue engineering (Huang et al., 2016). Jialong utilized the self-polymerization and reduction property

Front Pharmacol 12:759304.

doi: 10.3389/fphar.2021.759304 


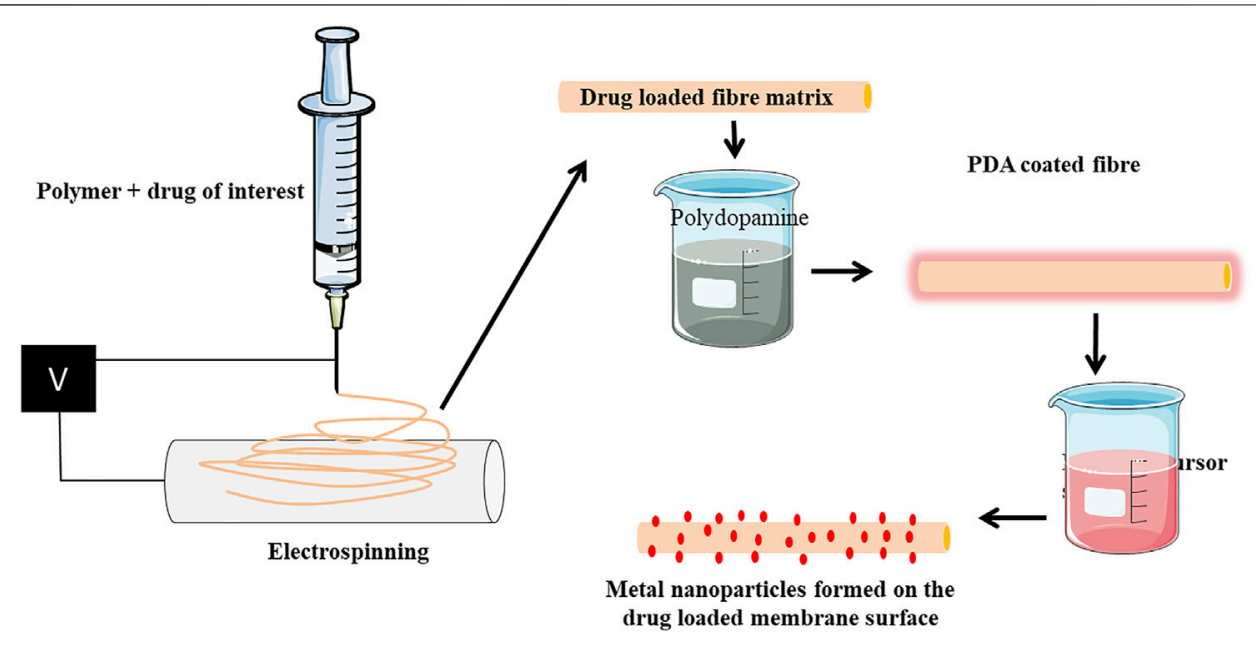

FIGURE 1 | Scheme representing the expansion of the ideology provided by Jialong et al. (2020). Drug can be incorporated into the PLLA fiber by mixing polymer and drug solution prior to electrospinning. The electron spun matrix can be coated with PDA followed by immersion into respective metal precursor solution. The membrane will have drug loaded into them and metallic nanoparticles will be formed on the surface. Such matrix can be used for various tissue engineering purposes for carrying drugs along with metallic nanoparticles.

of PDA and dip-coated PLLA@PDA membrane in silver nitrate solution to mediate silver nanoparticle formation. This musselinspired chemistry of PDA was previously shown to reduce the silver precursor- $\left[\mathrm{Ag}(\mathrm{NH} 3)_{2}\right]^{+}$ions to metallic silver nanoparticles, mediated by amine and cathecol groups ( $\mathrm{Wu}$ et al., 2015). Metal nanoparticles represent an emerging class of materials and known to regulate stem cell differentiation and osteoblast functions favoring bone formation. Gold, silver, iron, nickel, copper, strontium, selenium, and zirconium nanoparticles are known to regulate osteoblast functions (Suh et al., 2013; Zheng et al., 2014; Ye and Shi, 2018; Zhang et al., 2018; Eivazzadeh-Keihan et al., 2020; Tang et al., 2021). In addition to this, metal nanoparticles are known for their excellent antimicrobial properties. Antimicrobial resistance (AMR) is now a global concern halting the use of antibiotics and looking for alternative strategies. Metal nanoparticles offer promising hope in combating AMR. New antimicrobial chemistries are employed enabling the activation of antimicrobial functionality on demand without the need of antibiotics. Antimicrobial photodynamic therapy is of great interest in the recent past and an efficient alternative to the use of antibiotic-based therapies (Cieplik et al., 2018). For instance, inclusion of photosensitizers such as methylene blue (MB) and erythrosin B (ER) into PCL and PLGA electrospun membranes imparted visible light-dependent localized antimicrobial activity (Contreras et al., 2019). Photodynamic antimicrobial therapy is gaining considerable interests in the recent past. Very recently Carlos et al. reported the use of copper sulfide $(\mathrm{CuS})$ nanoparticles together with indocyanine green (ICG) as a synergistic approach to effectively induce antimicrobial activity against pathogenic bacterial and fungal species (Garin et al., 2021). Near-infrared light irradiation triggered the generation of intra and extracellular species, thereby killing the microbial cells. It is also note that the combination is cytofriendly to mammalian cells prompting its use for biomedical applications. One may note that, such nontoxic combinations can be used into various electrospun nanofibers and impart antimicrobial activity. In synchrony with such cytofriendly routes, the methods reported by Jialong et al. are devoid of any toxic reducing agents or complex instruments for this process. These metallic nanoparticles can be applied as such or loaded into nanofibrous scaffolds to mediate its osteogenic functions. Homogeneity if achieved, it can fill into the nanofibers and may provide an excellent surface area. Lower the loading content will result in less impact on the biocompatibility. To achieve optimal action, sustained release should be ensured which requires extensive loading of nanoparticles. This will adversely affect the stability of the fibrous matrix, mechanical properties, and thermal conductivity leading to faster degradation of the PLLA matrix. In addition to this, the provision for loading pharmacological agents targeting bone would be hindered. Therefore, we realized that the approach by Jialong et al., is expected to facilitate 1) in situ nanoparticles formation on the PLLA fibers without the involvement of external reducing agents and 2) loading of drugs into the PLLA fibers (Figure 1). Also, the wettability and surface hydrophilicity of PLLA can be improved. Additionally, we also speculate researchers can extend this study for coating biomedical implants. Electrospinning can be carried out on metallic implants (used as a collector) using PLLA/other polymers and dip coating in PDA followed by immersion in respective metal precursor solution will aid in formation of metallic nanoparticles. PDA alone is reported to adhere to various substrates to covalently immobilize numerous biomolecules owing to its copious reactive functional groups (Singh et al., 2021). The polar groups present in PDA not only aids in the formation of nanoparticles but also will support attachment and growth of cells (Qiu et al., 2018). Therefore, versatility of PDA-based surface modification is of much interest in orthopedic implants. 
There are some limitations in the present study. First, the size measurements of silver nanoparticles (AgNPs) formed on the surface is not reported. Silver nanoparticles are known to regulate cell functions and are size dependent. Silver nanoparticles at $50 \mathrm{~nm}$ were found to be cytotoxic compared to $3 \mu \mathrm{m}$ silver nanoparticles. The concentrations to induce antibacterial effects greater than that cause cytotoxic effects (Albers et al., 2013). AgNPs with the average size of $18 \mathrm{~nm}$ was found to cause apoptosis and necrosis (Zielinska et al., 2016). AgNPs accumulations also lead to ROS generation in osteoclasts. Therefore, studies on size measurements of silver or other metal nanoparticles produced by this route are needed to assess the potential health risks. Alarming data confirms the threat of silver nanoparticles on living organisms. Silver nanoparticles can enter into the natural habitat and hierarchy of trophic chain. It is known to disrupt cellular processes in plants and aquatic organisms (Bundschuh et al., 2018). AgNPs can enter into the animal respiratory system and causes pulmonary inflammation. In rats, it is accumulated in the liver, lungs, and induced genotoxicity (Pulit-Prociak and Banach, 2016). Therefore, careful considerations are mandatory for using AgNPs in healthcare sectors. Next, the biocompatibility was

\section{REFERENCES}

Albers, C. E., Hofstetter, W., Siebenrock, K. A., Landmann, R., and Klenke, F. M. (2013). In Vitro Cytotoxicity of Silver Nanoparticles on Osteoblasts and Osteoclasts at Antibacterial Concentrations. Nanotoxicology 7, 30-36. doi:10.3109/17435390.2011.626538

Arciola, C. R., Campoccia, D., Ehrlich, G. D., and Montanaro, L. (2015). Biofilmbased Implant Infections in Orthopaedics. Adv. Exp. Med. Biol. 830, 29-46. doi:10.1007/978-3-319-11038-7_2

Bundschuh, M., Filser, J., Lüderwald, S., McKee, M. S., Metreveli, G., Schaumann, G. E., et al. (2018). Nanoparticles in the Environment: Where Do We Come From, Where Do We Go to? Environ. Sci. Eur. 30 (1), 6-17. doi:10.1186/s12302018-0132-6

Cieplik, F., Deng, D., Crielaard, W., Buchalla, W., Hellwig, E., Al-Ahmad, A., et al. (2018). Antimicrobial Photodynamic Therapy - What We Know and what We Don't. Crit. Rev. Microbiol. 44 (5), 571-589. doi:10.1080/ 1040841X.2018.1467876

Contreras, A., Raxworthy, M. J., Wood, S., Schiffman, J. D., and Tronci, G. (2019). Photodynamically Active Electrospun Fibers for Antibiotic-free Infection Control. ACS Appl. Bio Mater. 2, 4258-4270. doi:10.1021/acsabm.9b00543

Cui, J., Yan, Y., Such, G. K., Liang, K., Ochs, C. J., Postma, A., et al. (2012). Immobilization and Intracellular Delivery of an Anticancer Drug Using Mussel-Inspired Polydopamine Capsules. Biomacromolecules 13, 2225-2228. doi:10.1021/bm300835r

Ding, Y. H., Floren, M., and Tan, W. (2016). Mussel-inspired Polydopamine for Bio-Surface Functionalization. Biosurf Biotribol 2, 121-136. doi:10.1016/ j.bsbt.2016.11.001

Eivazzadeh-Keihan, R., Bahojb Noruzi, E., Khanmohammadi Chenab, K., Jafari, A., Radinekiyan, F., Hashemi, S. M., et al. (2020). Metal-based Nanoparticles for Bone Tissue Engineering. J. Tissue Eng. Regen. Med. 14, 1687-1714. doi:10.1002/term.3131

Garin, C., Alejo, T., Perez-Laguna, V., Prieto, M., Mendoza, G., Arruebo, M., et al. (2021). Chalcogenide Nanoparticles and Organic Photosensitizers for Synergetic Antimicrobial Photodynamic Therapy. J. Mater. Chem. B 9 (31), 6246-6259. doi:10.1039/D1TB00972A

Hong, L., Liu, X., Tan, L., Cui, Z., Yang, X., Liang, Y., et al. (2019). Rapid Biofilm Elimination on Bone Implants Using Near-Infrared-Activated Inorganic Semiconductor Heterostructures. Adv. Healthc. Mater. 8, e1900835. doi:10.1002/adhm.201900835 performed by an indirect method using $48 \mathrm{~h}$ conditioned medium from PLLA@PDA membranes. This time point is not sufficient to predict the biocompatibility as it is dependent on 1) lactic and glycolic acids from PLLA and 2) silver nanoparticles form the PLLA surface. A longer time points must be considered to better predict the biocompatibility. Also, direct cell interaction studies on the construct will provide a better understanding on the toxicity. Therefore, when developing a PLLA construct coated with metallic nanoparticles mediated by PDA, one should consider the aforementioned factors in testing the biocompatibility.

\section{AUTHOR CONTRIBUTIONS}

Both SV and SS performed literature research, created the diagram, and wrote the manuscript.

\section{ACKNOWLEDGMENTS}

The authors thank the university management for the support.

Huang, S., Liang, N., Hu, Y., Zhou, X., and Abidi, N. (2016). Polydopamine-assisted Surface Modification for Bone Biosubstitutes. Biomed. Res. Int. 2016, 2389895. doi:10.1155/2016/2389895

Jiaolong, W., Linin, Z., Xianhua, Z., Runfa, Wu., Lan, L., and Junchao, W. (2020). Front. Pharmacol. 11, 431. doi:10.3389/fphar.2020.00431

Khatri, Z., Jatoi, A. W., Ahmed, F., and Kim, I.-S. (2016). Cell Adhesion Behavior of Poly(E-Caprolactone)/poly( L -lactic Acid) Nanofibers Scaffold. Mater. Lett. 171, 178-181. doi:10.1016/j.matlet.2016.02.061

Lee, H., Dellatore, S. M., Miller, W. M., and Messersmith, P. B. (2007). Musselinspired Surface Chemistry for Multifunctional Coatings. Science 318, 426-430. doi:10.1126/science.1147241

Liu, Y., Xu, C., Gu, Y., Shen, X., Zhang, Y., Li, B., et al. (2019). Polydopaminemodified Poly(l-Lactic Acid) Nanofiber Scaffolds Immobilized with an Osteogenic Growth Peptide for Bone Tissue Regeneration. RSC Adv. 9, 11722-11736. doi:10.1039/C8RA08828D

Mellonig, J. T., and Nevins, M. (1995). Guided Bone Regeneration of Bone Defects Associated with Implants: An Evidence-Based Outcome Assessment. Int. J. Periodontics Restorative Dent 15, 168-185.

Pulit-Prociak, J., and Banach, M. (2016). Silver Nanoparticles - A Material of the Future. . ?? Open Chem. 14, 76-91. doi:10.1515/chem-2016-0005

Qiu, W. Z., Yang, H. C., and Xu, Z. K. (2018). Dopamine-assisted Co-deposition: An Emerging and Promising Strategy for Surface Modification. Adv. Colloid Interf. Sci 256, 111-125. doi:10.1016/j.cis.2018.04.011

Ryu, J., Ku, S. H., Lee, H., and Park, C. B. (2010). Mussel-Inspired Polydopamine Coating as a Universal Route to Hydroxyapatite Crystallization. Adv. Funct. Mater. 20, 2132-2139. doi:10.1002/adfm.200902347

Singh, I., Dhawan, G., Gupta, S., and Kumar, P. (2021). Recent Advances in a Polydopamine-Mediated Antimicrobial Adhesion System. Front. Microbiol. 11, 3326. doi:10.3389/fmicb.2020.607099

Suh, K. S., Lee, Y. S., Seo, S. H., Kim, Y. S., and Choi, E. M. (2013). Effect of Zinc Oxide Nanoparticles on the Function of MC3T3-E1 Osteoblastic Cells. Biol. Trace Elem. Res. 155, 287-294. doi:10.1007/s12011-013-9772-y

Sun, X., Cheng, L., Zhao, J., Jin, R., Sun, B., Shi, Y., et al. (2014). bFGF-Grafted Electrospun Fibrous Scaffolds via Poly(dopamine) for Skin Wound Healing. J. Mater. Chem. B 2, 3636-3645. doi:10.1039/c3tb21814g

Tang, Y., Rajendran, P., Veeraraghavan, V. P., Hussain, S., Balakrishna, J. P., Chinnathambi, A., et al. (2021). Osteogenic Differentiation and Mineralization Potential of Zinc Oxide Nanoparticles from Scutellaria Baicalensis on Human Osteoblast-like MG-63 Cells. Mater. Sci. Eng. C Mater. Biol. Appl. 119, 111656. doi:10.1016/j.msec.2020.111656 
Trobos, M., Juhlin, A., Shah, F. A., Hoffman, M., Sahlin, H., and Dahlin, C. (2018). In Vitro Evaluation of Barrier Function Against Oral Bacteria of Dense and Expanded Polytetrafluoroethylene (PTFE) Membranes for Guided Bone Regeneration. Clin. Implant Dent Relat. Res. 20, 738-748. doi:10.1111/cid.12629

Willems, H. M., Xu, Z., and Peters, B. M. (2016). Polymicrobial Biofilm Studies: From Basic Science to Biofilm Control. Curr. Oral Health Rep. 3, 36-44. doi:10.1007/s40496-016-0078-y

Wu, C., Zhang, G., Xia, T., Li, Z., Zhao, K., Deng, Z., et al. (2015). Bioinspired Synthesis of polydopamine/Ag Nanocomposite Particles with Antibacterial Activities. Mater. Sci. Eng. C Mater. Biol. Appl. 55, 155-165. doi:10.1016/ j.msec.2015.05.032

Xie, M. M., Zhao, B. D., Wang, W. Y., Yu, L., and Zhang, X. Q. (2010). Effects of Oral Biofilm on Guided Bone Regeneration in Dental Implant. Chin. J. Tissue Eng. Res. 14, 2911. doi:10.3969/j.issn.1673-8225.2010.16.016

Ye, M., and Shi, B. (2018). Zirconia Nanoparticles-Induced Toxic Effects in Osteoblast-like 3T3-E1 Cells. Nanoscale Res. Lett. 13, 353-412. doi:10.1186/ s11671-018-2747-3

Zhang, X., Li, J., Wang, X., Wang, Y., Hang, R., Huang, X., et al. (2018). Effects of Copper Nanoparticles in Porous TiO2 Coatings on Bacterial Resistance and Cytocompatibility of Osteoblasts and Endothelial Cells. Mater. Sci. Eng. C Mater. Biol. Appl. 82, 110-120. doi:10.1016/j.msec.2017.08.061

Zheng, C., Wang, J., Liu, Y., Yu, Q., Liu, Y., Deng, N., et al. (2014). Functional Selenium Nanoparticles Enhanced Stem Cell Osteoblastic Differentiation
Through BMP Signaling Pathways. Adv. Funct. Mater. 24, 6872-6883. doi:10.1002/adfm.201401263

Zielinska, E., Tukaj, C., Radomski, M. W., and Inkielewicz-Stepniak, I. (2016). Molecular Mechanism of Silver Nanoparticles-Induced Human Osteoblast Cell Death: Protective Effect of Inducible Nitric Oxide Synthase Inhibitor. PLoS One 11, e0164137. doi:10.1371/journal.pone.0164137

Conflict of Interest: The authors declare that the research was conducted in the absence of any commercial or financial relationships that could be construed as a potential conflict of interest.

Publisher's Note: All claims expressed in this article are solely those of the authors and do not necessarily represent those of their affiliated organizations, or those of the publisher, the editors, and the reviewers. Any product that may be evaluated in this article, or claim that may be made by its manufacturer, is not guaranteed or endorsed by the publisher.

Copyright $\odot 2021$ Vimalraj and Sekaran. This is an open-access article distributed under the terms of the Creative Commons Attribution License (CC BY). The use, distribution or reproduction in other forums is permitted, provided the original author(s) and the copyright owner(s) are credited and that the original publication in this journal is cited, in accordance with accepted academic practice. No use, distribution or reproduction is permitted which does not comply with these terms. 